\title{
Importancia de la exploración médico forense en las agresiones sexuales a menores.
}

\author{
Importance of forensic medical exploration in the \\ sexual aggression of minors.
}

\section{M.L. Suárez Solá1 y F.J. González Delgado'}

\section{RESUMEN}

De un total de 58 denuncias por presuntas agresiones y abusos sexuales entre los años 1993 a 200I, las dividimos en tres grupos de edad (menores de 6 años, entre 6 y 12 años y de 12 a 18 años). Fueron estudiadas estadísticamente y examinados los hallazgos de las exploración física genital, extragenital y estado psíquico. En los resultados pudimos observar que denunciaron más las niñas mayores (13-18 años). Los hallazgos más sobresalientes fueron la integridad himeneal en el $76 \%$ de los casos, la exploración extragenital normal en el $83 \%$ y psicológica en el $74 \%$. Destacamos una dificultad para llegar al diagnóstico motivado por la ausencia de lesiones en la mayoría de los casos, lo que hace necesario que el examen físico y la toma de muestras se deba realizar lo antes posible. Hacemos hincapié en el valor terapéutico de una exploración física en las condiciones adecuadas ya que ayuda psicológicamente tanto a la presunta víctima como a los familiares próximos, refuerza el diagnóstico y puede reducir las secuelas futuras.

Palabras clave: violencia sexual, abusos sexuales, exploración física de menores, lesiones genitales.

\section{ABSTRACT}

We have registered a total of 58 incidents of alleged sexual aggression and abuse between 1993 and 2001 . They were categorized in three groups according to age (i.e., less than 6 years old, between 6 and 12 and between 13 and 18). We have carried out statistical studies on the samples and examined the results of genital and extra genital physical exploration as well as the mental state of the victim. The study shows that underage girls between 13 and 18 made the largest number of complaints to the police. The most significant finding was of hymeneal integrity in $76 \%$ of the cases, and normal extra genital and psychological exploration in $83 \%$ and $74 \%$ of the cases respectively. This shows the difficulty in proving the allegations in most of the cases due to the lack of physical evidence. It is, therefore, of paramount importance to perform a physical exploration and take medical samples as soon as possible. We would like to stress the therapeutic importance of a physical exploration under the right conditions, which provides psychological support to both the victim and the next of kin. It also helps to arrive at the proper diagnosis and to minimize any secondary effects that may occur.

Key words: sexual violence, sexual abuse, underage, physical exploration, genital lesions.

Correspondencia: María Luisa Suárez Solá. Clínica Médico Forense. Palacio de Justicia. Plaza del Adelantado. 382001 La Laguna (Santa Cruz de Tenerife). Tfno: 922208 071. FAX.: 922208070.

\footnotetext{
1 Doctor en Medicina y Cirugía, Especialista en Medicina Legal y Forense. Médico Forense Titular del Instituto de Medicina Legal de S/C. de Tenerife.
} 


\section{INTRODUCCIÓN:}

A pesar del enorme interés suscitado sobre los delitos sexuales en las niñas no hay muchas investigaciones en nuestra población. Además, hay que tener en cuenta que los casos denunciados, son simplemente el pico del iceberg. Son delitos que aparecen infrarepresentados en las denuncias (I).

Nos hemos propuesto valorar especialmente los reconocimientos físicos. En este trabajo estudiamos los datos propios que hemos obtenido durante los últimos ocho años de nuestro ejercicio profesional en Los Partidos Judiciales de Santa Cruz de Tenerife y de La Laguna, analizando el total de nuestras peritaciones a las que hemos aplicado diversas variables estadísticas.

La rapidez en el diagnóstico e identificación de las víctimas de abuso sexual es crucial para la reducción del sufrimiento de las niñas, por ello, es necesario establecer sistemas de apoyo para la asistencia, con el objetivo de que tengan un desarrollo físico y psicológico saludable o normal de adulta. Mientras las menores oculten el problema y la tiranía continúe, entonces el temor, el sufrimiento, la angustia y el secreto permanecerán con las víctimas (2, 3, 4 y 5).

Conocer lo que se espera en el examen puede ayudar a aliviar la ansiedad en el proceso. Para evitar preguntas repetidas innecesarias y potencialmente perjudiciales, especialmente en las menores, se realizará un historial detallado que debería suministrársele al médico forense previo a la evaluación.

El examen físico de las menores que han sufrido violencia sexual no debería resultar un trauma emocional adicional. Se le explicará a la niña en que consiste el examen antes de ejecutarlo. Es aconsejable que esté presente un acompañante de apoyo, no sospechoso, de estar involucrado en los hechos. Deberían realizarse exploraciones pediátricas a fondo, incluyendo unas breves valoraciones sobre el desarrollo, conducta, situación emocional y mental. Se pondrá atención especial al crecimiento y al desarrollo sexual. La técnica de estudio genital ha consistido habitualmente en un examen en posición supina con separación de labios y la posición genupectoral.

\section{PLANTEAMIENTOS Y OBjETIVOS.}

La Medicina Legal, Judicial o Forense, denominaciones todas ellas empleadas en lengua castellana, es el conjunto de conocimientos médicos y biológicos necesarios para la resolución de los problemas que plantea el Derecho, tanto en la aplicación práctica de las leyes como en su perfeccionamiento y evolución (6).

Nuestros objetivos y planteamientos son:

I. Analizar la información obtenida para alcanzar un mejor conocimiento.

2. Valorar la importancia de los hallazgos físicos que encontramos en las menores.

\section{MATERIAL Y MÉTODO:}

En nuestra práctica diaria médico-forense tenemos contactos con la violencia sexual, por este motivo hemos analizado los casos de menores hasta los 18 años, en los que hemos participado desde los años 1993 hasta el 2002 en los Partidos Judiciales de Santa Cruz (municipios de Santa Cruz de Tenerife y El Rosario) y de La Laguna (municipios de La Laguna, Tegueste, Tacoronte y el Sauzal).

Para la elaboración de nuestros resultados hemos dividido el conjunto de niñas estudiadas por presuntas agresiones sexuales y abusos sexuales en tres grandes grupos:

$$
\begin{aligned}
& \text { - Menores de } 6 \text { años, } \\
& \text { - entre } 6 \text { y } 12 \text { años y } \\
& \text { - de } 12 \text { a } 18 \text { años. }
\end{aligned}
$$

Recogimos para este trabajo un total de 58 denuncias. 


\section{RESULTADOS:}

De los casos expuestos hacemos una valoración recogiendo variables estadísticas y estudiando algunos indicadores que estimamos de utilidad para la valoración de la violencia sexual.

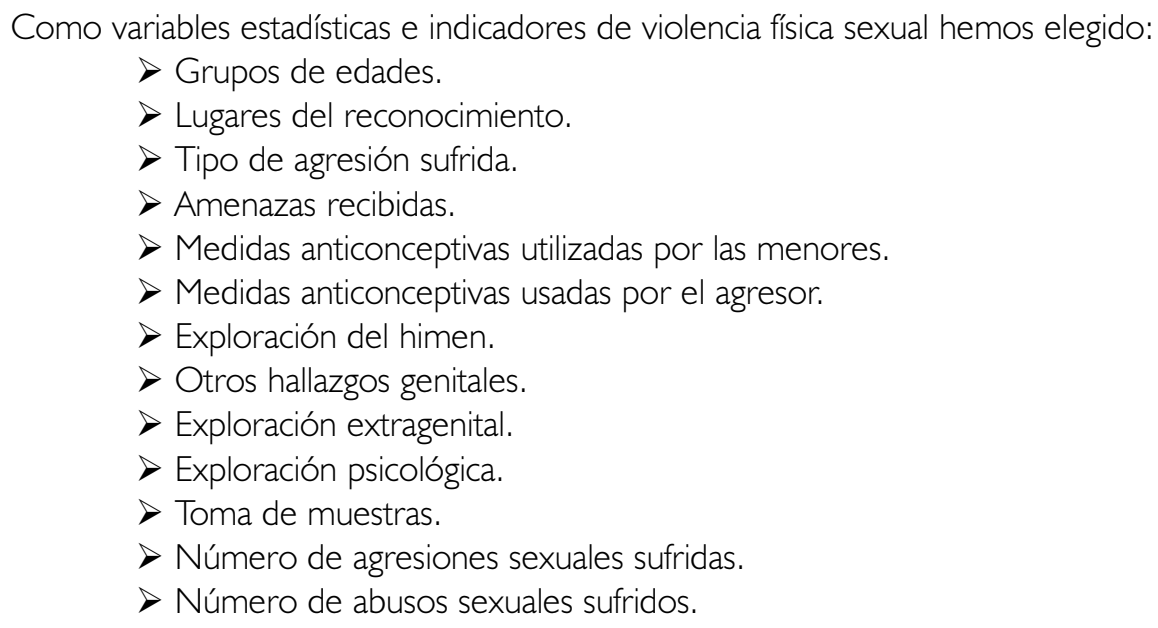

\section{GRUPOS DE EDADES:}

En nuestro estudio hemos dividido el total en tres grupos de edades de 6 años cada uno; en el primer grupo incluimos las niñas mas pequeñas (desde recién nacidas hasta los seis años de edad); el segundo grupo hasta la adolescencia (desde siete a doce años) y el tercero hasta la madurez (desde los trece a los 18 años). Observamos un mayor número de denuncias en este último grupo, con un total de 26 casos, seguido por las niñas prepúberes con 18 casos y las más pequeñas con I 4 casos (Tabla I).

\section{LUGAR DE RECONOCIMIENTO:}

Los reconocimientos fueron realizados en un centro hospitalario o en los Servicios Médicos Forenses de los Juzgados, al acudir directamente o al ser remitidos por la oficina de denuncias de la Policía. La exploración se practicó siempre por dos Médicos Forenses, o en compañía del Ginecólogo y Pediatra de guardia, cuando ha tenido lugar en un centro hospitalario. Como resultado en 19 ocasiones el lugar del reconocimiento fue en el Hospital Universitario de Canarias, frente a 9 casos en el Hospital Universitario de la Candelaria, y 16 en el Servicio Forense de Santa Cruz frente a 14 casos en el Servicio Forenses de La Laguna (Tabla 2).

\begin{tabular}{|l|c|}
\hline GRUPOS DE EDAD & N $^{\circ}$ DE CASOS. \\
\hline De 0 a 6 años & 14 \\
\hline De 7 a 12 años & 18 \\
\hline De 13 a 18 años & 26 \\
\hline
\end{tabular}

$\Delta$ TABLA 1

\begin{tabular}{|l|c|}
\hline LUGAR DE LA EXPLORACIÓN & N $^{\circ}$ DE CASOS \\
\hline Hospital Universitario de Canarias & 19 \\
\hline Servicio Forense de La Laguna & 14 \\
\hline Servicio Forense de Santa Cruz de Tenerife & 16 \\
\hline Hospital Univ. Ntra. Sra. de la Candelaria & 9 \\
\hline
\end{tabular}

$\triangle$ TABLA 2 


\section{TIPO DE AGRESIÓN SUFRIDA:}

La agresión más frecuentemente alegada fueron "solo tocamientos" con un total de 30 casos, seguidos de "solo penetración vaginal" con 14 casos. En un solo caso hubo intento de penetración vaginal. De los 10 casos de penetración anal, cuatro tuvieron además tocamientos, y en un caso hubo intento de penetración. En tres casos existió penetración vaginal y anal simultáneamente (Tabla 3).

\section{AMENAZAS RECIBIDAS:}

Casi la mitad de los casos estudiados, 28 niñas, no refieren ningún tipo de amenaza, y cuando existieron la mayoría fueron solo verbales, 17 casos, seguidas de amenazas con armas en 5 ocasiones, agresión sin armas en 4 y no especifican el tipo de amenazas 4 niñas (Tabla 4).

\begin{tabular}{||l|c||}
\hline TIPO DE AGRESIÓN & N $^{\circ}$ DE CASOS \\
\hline Tocamientos & 30 \\
\hline Intento de penetración anal & 1 \\
\hline Intento de penetración vaginal & 1 \\
\hline Penetración anal & 5 \\
\hline Tocamientos y penetración anal & 4 \\
\hline Penetración vaginal & 14 \\
\hline Penetración vaginal y anal & 3 \\
\hline
\end{tabular}

$\Delta$ TABLA 3

\begin{tabular}{||l|c||}
\hline \hline TIPO DE AMENAZA & $\mathbf{N}^{\circ}$ DE CASOS \\
\hline Ninguna & 28 \\
\hline Verbales & 17 \\
\hline No especifican & 4 \\
\hline Con armas & 5 \\
\hline Agresión sin armas & 4 \\
\hline
\end{tabular}

$\Delta$ TABLA 4

\section{MEDIDAS ANTICONCEPTIVAS UTILIZADAS POR LAS MENORES:}

Del total de 58 niñas estudiadas, el agresor no utilizó el preservativo ni se tomaron medidas barreras o anticonceptivas para prevenir infecciones o embarazos (Tablas 5 y 6 ).

\begin{tabular}{|l|c|}
\hline \multicolumn{1}{|c|}{ ANTICONCEPTIVOS } & NN $^{0}$ DE CASOS \\
\hline Medidas anticonceptivas & 0 \\
\hline Ninguna medida anticonceptiva & 58 \\
\hline
\end{tabular}

$\Delta$ TABLA 5

\begin{tabular}{||l|c|}
\hline TIPOS DE ANTICONCEPTIVOS & N $^{\circ}$ DE CASOS \\
\hline Preservativo & 0 \\
\hline OtroS & 0 \\
\hline
\end{tabular}

$\Delta$ TABLA 6

\section{EXPLORACIÓN DEL HIMEN:}

Realizado siempre con personal femenino, y en el grupo de menor edad acompañado de una persona de confianza. En la mayor parte de los casos, 4 I niñas, la exploración resultó negativa, no objetivándose lesiones; en ocho niñas se observaron desgarros antiguos. En seis niñas apreciamos desgarros recientes que se localizaron, siguiendo la localización horaria, a las 6 horas en tres niñas, a las 4 horas en dos y a las nueve horas en una niña. Por otro lado, en tres de las niñas se encontró como hallazgo una dilatación del himen que no correspondía con su edad (Tabla 7). 


\section{OTROS HALLAZGOS GENITALES:}

Aparte de las lesiones en el himen la exploración genital fue normal en la mayoría de las niñas (47); en dos niñas se apreció una pérdida de tono del esfínter anal; otras dos presentaron algunos eritemas y erosiones. Otros hallazgos observados de forma aislada fueron el exceso de flujo, eritema y eczema, erosión, eritema y excoriaciones, eritema y fisuras, excoriaciones en la comisura y desgarro en comisura con hematoma (Tabla 8).

\begin{tabular}{|l|c|}
\hline HALLAZGOS GENITALES & N$^{0}$ DE CASOS \\
\hline Normal, himen íntegro & 41 \\
\hline $\begin{array}{l}\text { Dilatación himeneal que no corresponde } \\
\text { a su edad }\end{array}$ & 3 \\
\hline Desgarros antiguos & 8 \\
\hline Desgarro reciente a las 6 horas & 3 \\
\hline Desgarro reciente a las 9 horas & 1 \\
\hline Desgarro reciente a las 4 horas & 2 \\
\hline
\end{tabular}

$\Delta$ TABLA 7

\begin{tabular}{|l|c|}
\hline OTROS HALLAZGOS GENITALES & N$^{\circ}$ DE CASOS \\
\hline Normal & 47 \\
\hline Flujo excesivo & 1 \\
\hline Pérdida de tono del esfínter anal & 2 \\
\hline Eritema y eczema & 1 \\
\hline Erosión & 1 \\
\hline Eritema y erosiones & 2 \\
\hline Eritema y excoriaciones & 1 \\
\hline Eritema y fisuras & 1 \\
\hline Excoriaciones en comisura & 1 \\
\hline $\begin{array}{l}\text { Desgarro en comisura y } \\
\text { hematoma }\end{array}$ & 1 \\
\hline
\end{tabular}

\section{EXPLORACIÓN EXTRAGENITAL:}

$\Delta$ TABLA 8

Fuera del área genital también las exploraciones fueron prácticamente negativas en la mayoría de las niñas (5 I casos). Los hallazgos que observamos de forma aislada fueron el eritema, hematoma perineal, contusión frontal, hematomas en antebrazos, hematomas en antebrazos y muslos, heridas cortantes y contusiones diversas (Tabla 9).

\section{EXPLORACIÓN PSICOLÓGICA:}

En 43 niñas no se llegaron a objetivar ninguna alteración psicológica que se pueda relacionar con la agresión sufrida. En 13 niñas el único signo clínico fue una elevación del nivel de nerviosismo o ansiedad; una niña estaba muy retraída y en otra solo se apreció una reducción parcial del nivel de conciencia (Tabla 10).

\section{EYACULACIÓN DEL AGRESOR:}

En más de la mitad de los casos el agresor no llegó a eyacular, en 32 casos frente a 26 en que sí lo hizo (Tabla II).

\section{TOMA DE MUESTRAS:}

El protocolo habitual persigue obtener muestras biológicas del agresor en la víctima o en el entorno donde ocurrieron los hechos. No obstante, atendiendo a las circunstancias, en la mayoría de las ocasiones (43 niñas) no fue necesario tomar muestras, ante la imposibilidad de encontrar hallazgos; en I3 de las niñas se tomaron muestras de secreciones en genitales, de las ropas y una toma de sangre; en una ocasión solo en genitales y ropa, y en una niña solo se tomaron muestras a nivel vaginal (Tabla 12). 


\begin{tabular}{|l|c||}
\hline \hline HALLAZGOS EXPLORACIÓN EXTRAGENITAL & N $^{0}$ DE CASOS \\
\hline Normal, sin lesiones & 51 \\
\hline Eritema & 1 \\
\hline Hematoma perineal & 1 \\
\hline Contusión frontal & 1 \\
\hline Hematomas en antebrazos & 1 \\
\hline Hematomas en antebrazos y muslos & 1 \\
\hline Heridas cortantes & 1 \\
\hline Contusiones diversas & 1 \\
\hline \hline
\end{tabular}

\begin{tabular}{|l|c|}
\hline $\begin{array}{l}\text { HALLAZGOS EXPLORACIÓN } \\
\text { PSICOLOGICA }\end{array}$ & No DE CASOS \\
\hline Nerviosismo. & 13 \\
\hline Retraída. & 1 \\
\hline Ligera obnubilación. & 1 \\
\hline Sin alteraciones aparentes. & 43 \\
\hline
\end{tabular}

$\Delta$ TABLA 10

$\Delta$ TABLA 9

\begin{tabular}{|c|c|}
\hline EYACULACIÓN DEL AGRESOR & Nº DE CASOS \\
\hline Sí & 26 \\
\hline No & 32 \\
\hline
\end{tabular}

\begin{tabular}{|l|c|}
\hline \multicolumn{1}{|c|}{ TIPO DE MUESTRAS } & No DE CASOS \\
\hline No fue necesario & 43 \\
\hline Genitales, sangre y ropas & 13 \\
\hline Genitales y ropa & 1 \\
\hline Vaginales & 1 \\
\hline
\end{tabular}

$\Delta$ TABLA 12

$\Delta$ TABLA 11

\section{DISCUSIÓN:}

\section{- Evaluación de los reconocimientos.}

Antes de evaluar la relevancia de los reconocimientos físicos y psíquicos debemos señalar la importancia de los mismos, a pesar de que, en algunas ocasiones, las víctimas no desean ser reconocidas, lo cual dificulta la valoración. Es conveniente que el reconocimiento se realice en presencia de una mujer o que el explorador sea de este sexo, ya que facilita mucho el examen, debido a que con frecuencia se rechaza al sexo masculino, al ser el agresor habitualmente un hombre, viéndose reflejado, en algunos casos, el delincuente con el examinador. También es importante en la exploración poder suministrar seguridad y tranquilidad, especialmente psíquica, tanto a la menor como a su familia, pero en ocasiones, sus propias reacciones mentales le impiden cooperar, lo que hace más laboriosa la investigación.

El diagnóstico de una agresión sexual o abuso también es difícil, debido a que con frecuencia puede estar basado solo en la historia de la niña. La experiencia nos ha enseñado que se necesitan muchos estudios de investigación para entender la anatomía normal femenina prepuberal e interpretar las conclusiones en los casos de violencia sexual. Una abertura vaginal mayor de $5 \mathrm{~mm}$ 
no es común y puede indicar la penetración vaginal con un dedo, un objeto o el pene. (7). Otro problema que dificulta el diagnóstico es el hecho de que los signos físicos están con frecuencia ausentes, incluso cuando el perpetrador del atropello admite la penetración en los genitales de la niña. En nuestros resultados no encontramos habitualmente lesiones, coincidiendo con otros autores, que aún utilizando métodos novedosos, como una exploración fotográfica con colposcopio, tampoco han observado hallazgos positivos (8). Hay que tener en cuenta que muchos tipos de forzamientos no dejan evidencia física y que las heridas de la mucosa curan muy rápido por lo que el diagnóstico precisaría de un reconocimiento precoz. En ocasiones se presentan dificultades incluso en los casos que muestran una clara evidencia de un trauma anogenital pero en los que la menor niega los hechos o, por otro lado, hay hallazgos que si bien están relacionados con abusos, de forma aislada no son suficientes para llegar a su diagnóstico.

El forense y el equipo multidisciplinar que evalúa a la niña deben establecer un nivel de certeza acerca de si una niña ha sufrido o no un atropello sexual.

La presencia de semen, esperma o fosfatasa ácida; un cultivo positivo de gonorrea; o una prueba serológica positiva, para la infección por síflis o el virus de inmunodeficiencia humana (HIV), le dan al diagnóstico de violencia sexual una evidencia médica, incluso en la ausencia de un historial positivo, siempre y cuando se excluyan las formas congénitas de gonorrea, śfilis y HIV adquirido por transfusión o de otra forma. No obstante, la mayoría de las veces no se pueden recoger muestras de semen, entre otros factores por haber transcurrido mucho tiempo, no existir eyaculación o porque esta ha ocurrido por fuera del cuerpo de la menor y no saber indicarlo. En nuestro estudio la mayor parte de las veces no ha sido posible recoger vestigios por estos motivos y en otras ocasiones porque se trata de tocamientos con el pene.

Un punto que hay que conocer es que ninguna niña está psicológicamente preparada para saber sobrellevar repetidas estimulaciones sexuales. Incluso a edades muy cortas, de dos o tres años, cuando no conocen lo que es la actividad sexual. Es "erróneo" pensar que no desarrollarán problemas en un futuro. En nuestro estudio pudimos observar que las niñas de cinco o más años de edad que conocen al autor del abuso se preocupan por él, quedando atrapadas por el afecto o la lealtad al agresor. Estas menores tienen la sensación de que las actividades sexuales son terriblemente malas. Si la menor trata de romper la relación sexual, el perpetrador del atropello puede amenazar a la niña con violencia o con la de pérdida del cariño que para la menor es muy importante. Cuando ocurre la violencia sexual dentro de la familia, puede temer el enfado, los celos, la lástima de los otros miembros de su casa, y la angustia de que su hogar se rompa si se revela el secreto. En nuestras víctimas de abuso sexual prolongado encontramos una baja autoestima, un sentimiento importante de culpabilidad y una visión distorsionada del sexo, al igual que la mayoría de los estudios realizados $(9,|0 \mathrm{y}| \mathrm{I})$.

La exploración debe ser inmediata, incluso antes de realizar la denuncia, teniendo la ventaja de poder auxiliar a la policía en su investigación y acudir así posteriormente al juicio con los datos suficientes y con más fiabilidad. El sistema actual no permite un seguimiento total a lo largo del tiempo, sino que acaba cuando se le da el alta, pero sabemos que el juicio produce el recuerdo de todo lo sucedido, pudiendo provocar alteraciones psíquicas que se deben también evaluar. A este respecto, se están realizando estudios como los de Price y cols. (I2), debiendo extenderse la continuidad de la asistencia psicológica de la menor no solo hasta el juicio, sino posterior a él, debido a que en la mayoría de las ocasiones persisten secuelas que precisan ayuda o apoyo psicológico. 


\section{- El valor de los hallazgos físicos.}

Debemos tener en cuenta que la organización y la técnica del examen juegan un papel importante. La exploración debe realizarse en una habitación tranquila, tomándose el tiempo que sea necesario, en ocasiones huyendo del formalismo, creando un clima de confianza y seguridad con el explorador/a. Se estudiará toda la documentación de forma meticulosa, posteriormente se practicará el examen que requerirá de una iluminación adecuada, y por último, pero no de menor importancia, es la cualificación del personal (13), de ahí la necesidad de un equipo de forenses especializados en las agresiones sexuales y con una buena disposición para encontrar los signos de la evidencia.

Con frecuencia los hallazgos positivos dependen principalmente del tipo de abuso, del área anogenital que resultó afectada y del grado de fuerza usada. Como hemos referido, muchas formas de violencia sexual no dejan ningún rastro ( 14 ) y es muy raro que se use fuerza física importante, como lo reflejan nuestros resultados. Es también un factor primordial el tiempo transcurrido desde el atentado hasta que se realiza la exploración. Generalmente suele ser de varios días, meses o años, dependiendo de cuando la menor, la familia u otros decidan delatar los hechos. En nuestra experiencia, en nuestra población, la proporción de denuncias es baja.

Los indicios recientes son más fáciles de evaluar que los crónicos, pero las pequeñas violentadas raramente se presentan en la situación aguda (excepto cuando los perpetradores son desconocidos). Los signos físicos con un significado forense ocurren raramente y por lo general solo se encuentran hasta 72 horas después de la agresión.

No encontramos casi lesiones físicas en las menores exploradas, estando el himen íntegro en un $76 \%$ de las niñas examinadas, sin otras señales de violencia genital ni extragenital, y cuando se presentan heridas, erosiones o contusiones estas suelen ser leves. En este trabajo de investigación, el hallazgo físico más frecuente en el área genital ha sido el eritema, asociado a eczema, erosiones o excoriaciones. No obstante, se debe tener en cuenta que este eritema puede ser un hallazgo "normal" que se encuentra en niñas no abusadas en un 56\% (I5).

A pesar de que en nuestros resultados no hemos observado enfermedades infecto-contagiosas constatadas, las verrugas y herpes genitales se pueden transmitir en el abuso sexual y deben buscarse ( 16 ).

Como hemos dicho, no solemos encontrar evidencias físicas, y en las exploraciones psiquiátricas tampoco objetivamos alteraciones evidentes inmediatas, pero debemos señalar, que no podemos realizar el seguimiento suficiente para evaluar la repercusión real en el desarrollo psíquico de estas menores, ya que sabemos que pueden tener problemas de adultas, especialmente sentimientos de baja autoestima, de culpa y dificultades en sus relaciones con otras personas que pueden repercutir en sus vida futura. También hemos observado que tiene mucha importancia el número de agresiones sufridas siendo mayor la repercusión cuando el perpetrador ha sido el propio progenitor o familiar, añadiéndose así problemas económicos y familiares, tales como sentirse rechazada por algún miembro de su hogar.

A nivel judicial y forense, puede ser habitual que solo se valore el hecho en sí y a la víctima de forma aislada, pero la exploración judicial en las menores debe ir más allá, valorando psíquicamente a las demás personas implicadas.

\section{CONCLUSIONES:}

I. La violencia sexual en las niñas es más frecuente en el grupo de edad de los 13 a 18 años.

2. La edad más frecuente son los dieciséis años, habiendo también picos significativos a los 5 y 9 años. 
3. Se suele realizar el primer reconocimiento forense en los Centros Hospitalarios.

4. El tipo de agresión sexual más frecuente son los tocamientos.

5. En un $48 \%$ no hubo amenazas.

6. No utilizan anticonceptivos femeninos.

7. En ningún caso el agresor utilizó preservativo.

8. El himen estaba íntegro en el $76 \%$.

9. No habían otras lesiones genitales en el $84 \%$.

10. La exploración extragenital fue normal en el $87 \%$.

I I. La exploración psicológica fue normal en el $74 \%$.

12. No fue posible tomar muestras en el $74 \%$.

\section{BIBLIOGRAFÍA:}

I. SIMPSON K. Medicina Forense. Espaxs, Zaragoza; 198I. 244.

2. BAGLEY C. Development of an adolescent stress scale for use of school counsellors. School Psychology International, 1992; 13, 31-49.

3. BAGLEY C. The prevalence and mental health sequels of child sexual abuse in a community simple of women aged 18 to 27. Canadian Journal of Community Mental Health; 199|; 10, 103-116. 4. FINKELHOR D, HOTALING G, LEWIS IA, SMITH C. Sexual abuse in a national survey of adult men and women prevalence, characteristics and risk factors. Child Abuse and Neglect, 1990; 14, 19-28. 5. WHITLOCK K, \& GILLMAN R,. Sexuality: a neglected component of child sexual abuse education and training. Child Welfare, 1989; 68, 317-29.

6. GISBERT CALABUIG JA. Medicina Legal y Toxicología. $5^{\text {a }}$ ed. Masson. Barcelona; 1998; 3.

7. HERRMANN-GIDDENS MF \& FROTHINGHAM TE. Prepubertal female genitalia: examination for evidence of sexual abuse. Paediatrics.1987; 80(2):203-8.

8. ADAMS JA, HARPER K, KNUDSON S, REVILLA J.. Examination findings in legally confirmed child sexual abuse it 's normal to be normal. Paediatric. 994; 97(I):148-50.
9. FINKELHOR D. The trauma of child abuse: Two models. En $G$ Wyatt y $G$ Powell (Ed): Lasting effects of child sexual abuse. London: Sage Publications. 1979; 1988.

10. FINKELHOR D \& ARAJI S. Explanations of pedophilia: A four factor model. Journal of Sex Research. 1986; 22(2):145-161.

II. MALTZ W. Identifying and testing the sexual repercussions of incest. A couple therapy approach. Journal of Sex and Marital Therapy. 1988; 14(2): I-35.

12. PRICE JL, HILSENROTH MJ, PETRETIC-JACKSON PA, BONGE D. A review of individual psychotherapy outcomes for adult survivors of childhood sexual abuse. Clin Psychol Rev. 2001;21(7):1095-121.

13. HERRMANN B, VIET S, NEISES M. Medical diagnosis in child sexual abuse. Official Journal of the German Paediatric Society. Monatsschrift Kinderheilkunde 1997; 145: 1219-1226.

14. BAYS I \& CHADWICK D. Medical Diagnosis of the sexually abused child. Chid Abuse Negl. 1993; 17:91-107.

15. MCCANN J, WELLS R, SIMON M, VORIS J. Genital findings in prepubertal girls selected for no abuse: a descriptive study. Paediatrics 1990; (3):428-39.

16. FISCHER GO. Vulval disease in pre-pubertal girls. Australia J Dermatol 2001; 42(4);225-36. 